\title{
Improved Face Recognition with Multilevel BTC using Kekre's LUV Color Space
}

\author{
H.B. Kekre, \\ Senior Professor \\ Computer Engineering Department \\ MPSTME, SVKM's NMIMS \\ Mumbai, India
}

\author{
Dr. Sudeep Thepade \\ Associate Professor \\ Computer Engineering Department \\ MPSTME,SVKM's NMIMS \\ Mumbai,India
}

\author{
Sanchit Khandelwal, Karan \\ Dhamejani, Adnan Azmi, \\ B.tech Students \\ Computer Engineering Department \\ MPSTME, SVKM's NMIMS \\ Mumbai, India
}

\begin{abstract}
The theme of the work presented in the paper is Multilevel Block Truncation Coding based Face Recognition using the Kekre's LUV (K'LUV) color space. In [1], Multilevel Block Truncation Coding was applied on the RGB color space up to four levels for face recognition. The experimental results showed that Block Truncation Coding Level 4 (BTC-level 4) was better as compared to other BTC levels of RGB color space. Results displaying a similar pattern are realized when the K'LUV color is used. It is further observed that K'LUV color space gives improved results on all four levels.
\end{abstract}

Keywords- Face recognition; BTC; RGB; K'LUV; Multilevel BTC; FAR; GAR.

\section{INTRODUCTION}

The term face recognition refers to identifying and verifying a face image. It is basically the process of classifying a face as 'known' or 'unknown', based on training set. While humans can easily identify faces, it is a challenging task for computer systems. The computer systems store the faces in such way that the important contents of the face image they store, can be used efficiently for recognizing the face.

There are many biometric systems such as finger prints, voice, iris, face and retina. Among these face recognition turns out to be the most effective system since it requires very less human interaction [21, 22]. Researchers from the field of biometrics, image processing, computer vision, pattern recognition system and neural network give a lot of importance to face recognition. It is the fastest growing biometric technology [18]. Some of the applications of face recognition include physical, security and computer access controls, law enforcement [12, 13], criminal list verification, surveillance at various places [15], forensic, authentication at airports[17], etc.

Many algorithms are used to make effective face recognition systems. Some of the algorithms include Principle Component Analysis (PCA) [2, 3, 4, 5], Linear Discriminant Analysis (LDA) [6, 7, 8], Independent Component Analysis (ICA) $[9,10,11]$, Block Truncation Coding (BTC) [1, 15, 19, 22] etc.

The paper presents an approach to enhance the performance of BTC based face recognition using K'LUV color space.
Applying the technique described in [1], using K'LUV color, it is observed that K'LUV out performs RGB color space at each level of Multilevel BTC.

\section{Block TRUnCATION CODING}

Block truncation coding (BTC) [1, 12, 13, 14] was developed in the early years of digital imaging more than 29 years. It was first developed in 1979 for greyscale image coding [14]. It is comparatively a simple image coding technique. BTC has played a vital role in the history of digital image coding in such a way that many advanced coding techniques have been developed, based on BTC.

\section{Multilevel Block Truncation Coding [1, 13, 20]}

The feature vector in this algorithm is calculated by using Block Truncation Coding [12, 13 and 14]. In [1], BTC has been implemented up to four levels on RGB colour space for face recognition. The feature vector size at BTC-Level 1, BTCLevel 2, BTC-Level 3 and BTC-Level 4 was 6, 12, 24 and 48 respectively. In the same way BTC on K'LUV colour space is implemented up to four levels for face recognition.

\section{KeKRE's LuV COLOR SPACE}

It was obvious to extend BTC to multi-spectral images such as color images. Most color images are recorded in RGB space, which is perhaps the most well-known color space.

K'LUV color space [12] is a special case of Kekre transform. Where $\mathrm{L}$ gives luminance and $\mathrm{U}$ and $\mathrm{V}$ gives chromaticity values of color image. Positive value of $U$ indicates prominence of red component in color image and negative value of $\mathrm{V}$ indicates prominence of green component.

Equation (1) gives the RGB to LUV conversion matrix which indicates the corresponding $\mathrm{L}, \mathrm{U}$ and $\mathrm{V}$ components for an image from the $\mathrm{R}, \mathrm{G}$ and $\mathrm{B}$ components.

$$
\left[\begin{array}{l}
L \\
U \\
V
\end{array}\right]=\left[\begin{array}{ccc}
1 & 1 & 1 \\
-2 & 1 & 1 \\
0 & -1 & 1
\end{array}\right]\left[\begin{array}{l}
R \\
G \\
B
\end{array}\right]
$$

The reverse conversion, that is from LUV color space to RGB color space is given in Equation (2). 


$$
\left[\begin{array}{l}
R \\
G \\
B
\end{array}\right]=\left[\begin{array}{ccc}
1 & -2 & 0 \\
1 & 1 & -1 \\
1 & 1 & 1
\end{array}\right]\left[\begin{array}{l}
L / 3 \\
U / 6 \\
V / 2
\end{array}\right]
$$

\section{PROPOSED METHOD}

For color space, for each BTC level; the feature vector for the query image and database set is by using Multilevel BTC.

In each level of BTC, the feature vector of the query image is compared with the feature vector of each image in the training set. The comparison (Similarity measurement) is done by Mean Square Error (MSE) given by equation 3 .

$$
\operatorname{MSE}=\frac{1}{\operatorname{MN}} \sum_{\mathrm{y}=1}^{\mathrm{M}} \sum_{\mathrm{x}=1}^{\mathrm{N}}\left[\mathrm{I}(\mathrm{x}, \mathrm{y})-\mathrm{I}^{\prime^{\prime}, \prime^{\prime \prime}}{ }^{\prime}(\mathrm{x}, \mathrm{y})\right]^{2}
$$

Where,

I \& I' are two feature vectors of size $\mathrm{M}^{*} \mathrm{~N}$ which are being compared.

To assess the performance of the different BTC levels based face recognition techniques, False Acceptance Rate (FAR) and Genuine Acceptance Rate (GAR) are used.

\section{IMPLEMENTATION}

\section{A. Platform}

The effectuation of the Multilevel BTC is done in MATLAB 2010. It was carried out on a computer using an Intel Core i5-2410M CPU (2.4 GHz).

\section{B. Database}

The experiments were performed on two face databases.

\section{1) Face Database [16]}

This database is created by Dr Libor consisting of 1000 images (each with 180 pixels by 200 pixels), corresponding to 100 persons in 10 poses each, including both males and females. All the images are captured against a dark or bright homogeneous background, little variation of illumination, different facial expressions and details. The subjects sit at fixed distance from the camera and are asked to speak, whilst a sequence of images is taken. The speech is used to introduce facial expression variation. The images were taken in a single session. The ten poses of Face database are shown in Figure 1.

\section{2) Our Own Database [1,20]}

This database consists of 1600 face images of 160 people (92 males and 68 females).For each person 10 images are taken. The images in the database are captured under numerous illumination settings. The images are taken with a homogenous background with the subjects having different expressions. The images are of variable sizes, unlike the Face database. The ten poses of Our Own Database are shown in Figure 2.

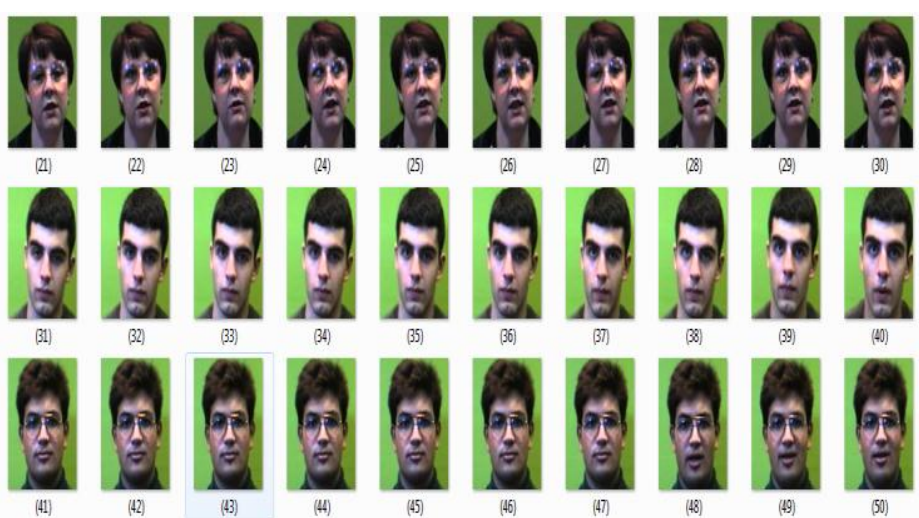

Figure 1. Sample images from Face database

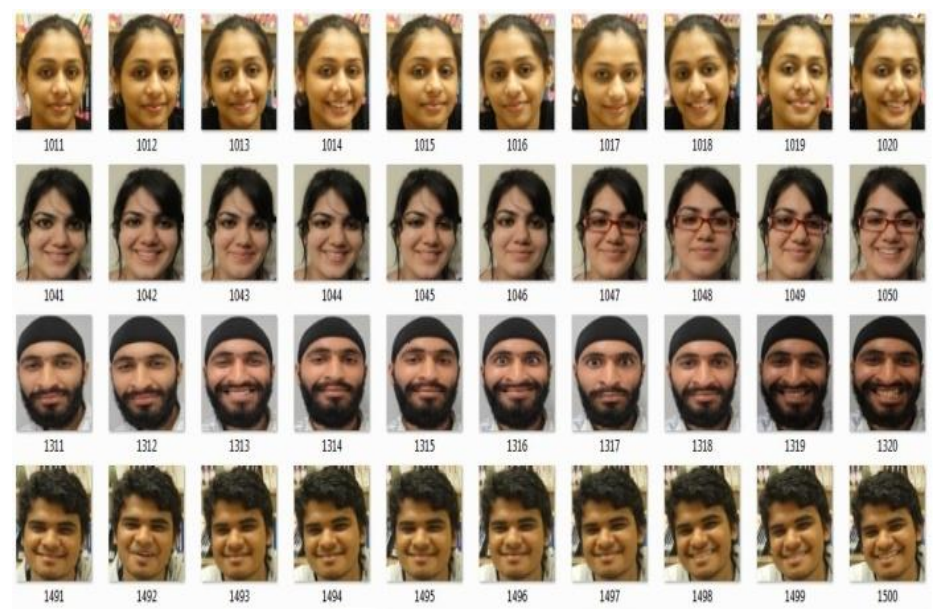

Figure 2. Sample images from Our Own Database

\section{ResUlts AND Discussions}

False Acceptance Rate (FAR) and Genuine Acceptance Rate (GAR) are standard performance evaluation parameters of face recognition system.

The False acceptance rate (FAR) is the measure of the likelihood that the biometric security system will incorrectly accept an access attempt by an unauthorized user. A system's FAR typically is stated as the ratio of the number of false acceptances divided by the number of identification attempts. 100

$\mathrm{FAR}=($ False Claims Accepted/Total Claims $) \mathrm{X}$

The Genuine Acceptance Rate (GAR) is evaluated by subtracting the FAR values from 100 .

$$
\text { GAR=100-FAR (in percentage) }
$$

In all 10000 queries (10 images for each of 1000 persons) are fired on face database and 16000 queries (10 images for each of 1600 persons) are fired on our own database. For each query, FAR and GAR values are calculated for respective BTC level based face recognition technique. At the end the average FAR and GAR of all queries in respective face databases are considered for performance ranking of BTC levels based face recognition techniques. 
FAR and GAR are calculated for both RGB color space and K'LUV color space.

\section{A. Face Database}

To analyze the performance of proposed algorithm, 10000 queries are tested on the database. The feature vectors of each image for all four BTC levels in RGB color space and K'LUV color space were calculated and then compared with the database. The FAR and GAR values are calculated by employing equations 4 and 5 .

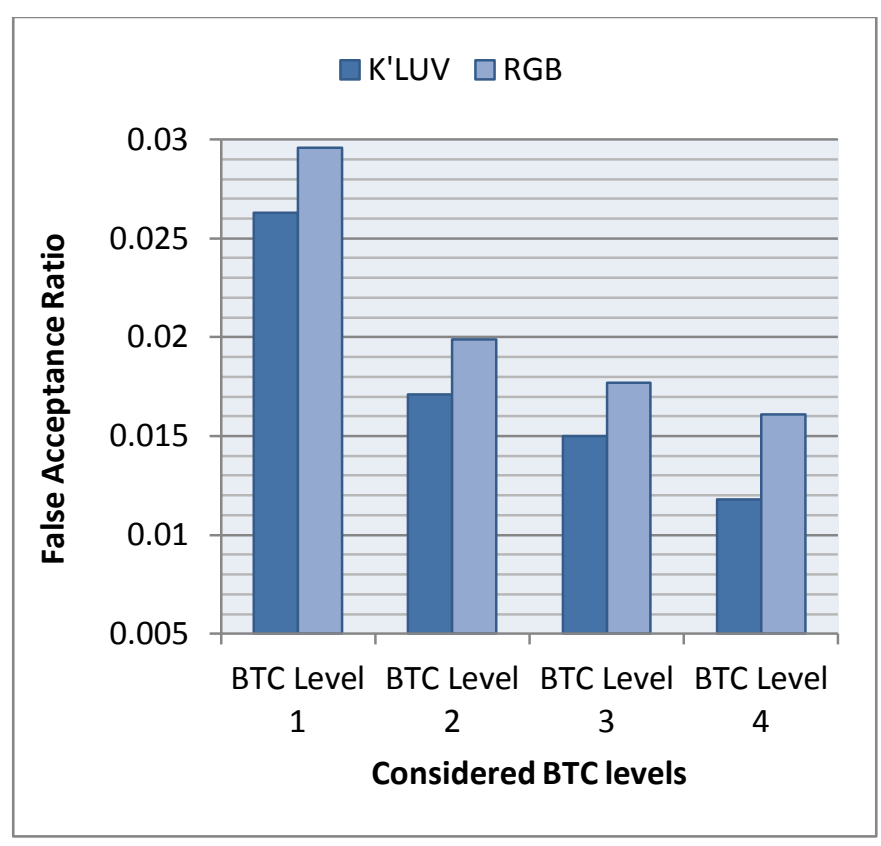

Figure 3. FAR values at different BTC levels of K'LUV and RGB color spaces for Face Database

Figure 3 gives the FAR values of the different BTC levels based face recognition techniques tested on face database for both RGB and K'LUV color spaces. Here it can be seen that the FAR values go on decreasing for each succeeding level of BTC of respective color spaces. This shows that the accuracy of face recognition increases with increasing level of BTC and hence BTC-level 4 gives the best result with the least FAR value in both the color spaces. Also the FAR values of K'LUV are lesser than the RGB as shown in the figure. Thus, it can be concluded that the implementation of BTC levels based face recognition techniques is better when applied in K'LUV color space.

Figure 4 gives the GAR values of the different BTC levels based face recognition techniques tested on face database for both RGB and K'LUV color spaces. Here it is observed that with each successive level of BTC the GAR values go on increasing in respective color spaces and hence a BTC-level 4 gives the best result with the highest value in both the color spaces. Also the GAR values of K'LUV are greater than the RGB as shown in the figure. Thus, it can be concluded that the implementation of BTC levels based face recognition techniques is better when applied in K'LUV color space. For optimal performance the FAR values must be less and accordingly the GAR values must be high for each successive levels of BTC. Thus, the performance of K'LUV color space for BTC levels based face recognition techniques is superior to the performance of RGB color space for Face database.

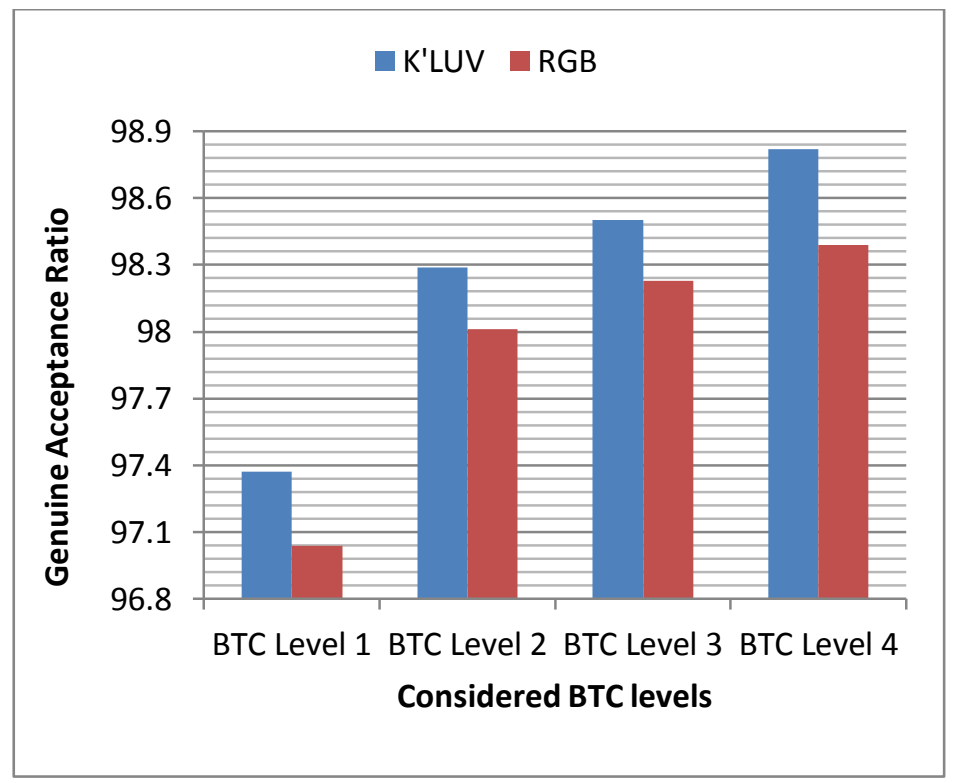

Figure 4. GAR values at different BTC levels of K'LUV and RGB color spaces for Face Database

\section{B. Our Own Database}

In all 16000 queries were tested on the database for analyzing the performance of the proposed BTC level based face recognition algorithm for both RGB color space and K'LUV color space. The experimental results of proposed face recognition techniques have shown that BTC level 4 gives the best performance in respective color spaces. The efficiency of the Multi-level BTC based face recognition increases with the increasing levels of BTC.

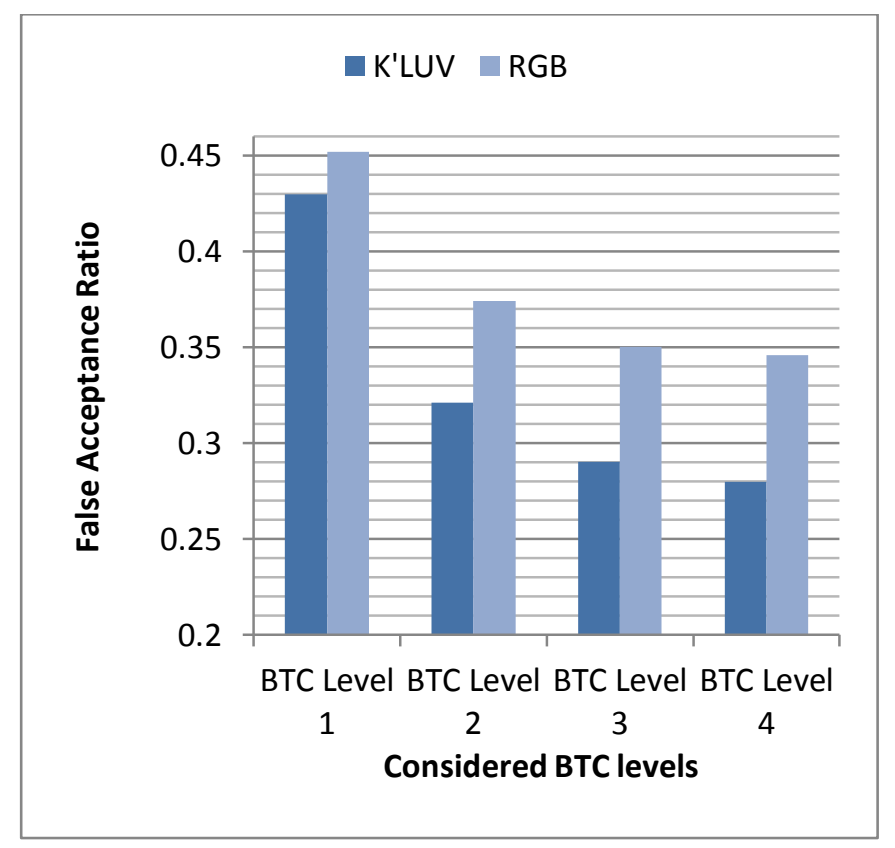

Figure 5. FAR values at different BTC levels of K'LUV and RGB color spaces for Our Own Database 
Figure 5 gives the FAR values of the different BTC levels based face recognition techniques tested on Our Own Database for both RGB and K'LUV color spaces. The FAR values go on decreasing for each succeeding level of BTC of respective color spaces. Thus, when BTC based face recognition techniques is applied on Our Own Database, it gives a result similar to the Face Database; The BTC level 4 gives the best result for respective color spaces and K'LUV color space is better than RGB color space for implementing this proposed algorithm.

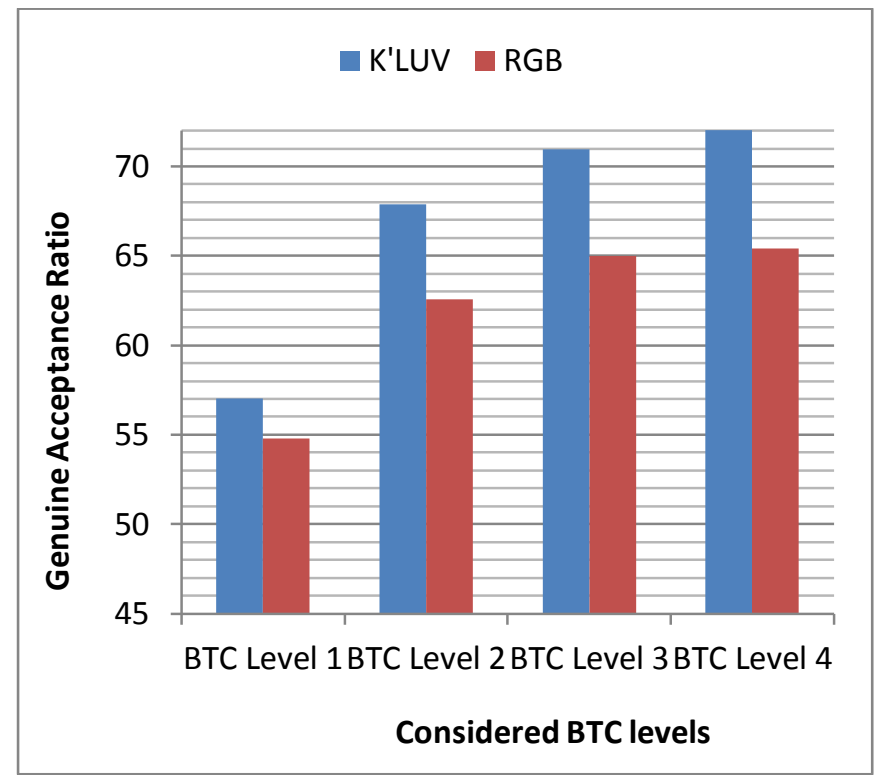

Figure 6. GAR values at different BTC levels of K'LUV and RGB color spaces for Our Own Database

Figure 6 gives the GAR values of the different BTC levels based face recognition techniques tested on Our Own Database for both RGB and K'LUV color spaces. It can be seen from the above figure that BTC-Level 4 has the highest GAR values and hence it is better than other BTC-Levels. Also the GAR values of K'LUV color space are greater than RGB color space at all the levels. Thus it can be concluded that the implementation of BTC levels based face recognition techniques is better when applied in K'LUV color space.

As seen from the performance of both the databases it can be concluded that the implementation of BTC based face recognition techniques on K'LUV color space is superior to RGB color space.

\section{CONCLUSION}

As the Multilevel BTC yields a greatly reduced feature space, this reduces the processing time required by the system. Thus, this system can be implemented is real time applications which generally require fast recognition time and have low computation power. The proposed face recognition system using Multilevel BTC has been tested using two face databases. For experimental analysis in all 10000 queries are fired on Face Database and 16000 queries on Our Own Database. The average FAR and GAR values of these queries clearly indicate that better performance is obtained when Multilevel Block
Truncation Coding is employed using Kekre's LUV color space than RGB color space for face recognition.

\section{REFERENCES}

[1] H.B.Kekre, Sudeep D. Thepade, Sanchit Khandelwal, Karan Dhamejani, Adnan Azmi, "Face Recognition using Multilevel Block Truncation Coding" International Journal of Computer Applications (IJCA) December 2011 Edition.

[2] Xiujuan Li, Jie Ma and Shutao Li 2007. A novel faces recognition method based on Principal Component Analysis and Kernel Partial Least. IEEE International Conference on Robotics and Biometrics, 2007. ROBIO 2007

[3] Shermin J "Illumination invariant face recognition using Discrete Cosine Transform and Principal Component Analysis" 2011 International Conference on Emerging Trends in Electrical and Computer Technology (ICETECT).

[4] Zhao Lihong, Guo Zikui "Face Recognition Method Based on Adaptively Weighted Block-Two Dimensional Principal Component Analysis"; 2011 Third International Conference on Computational Intelligence, Communication Systems and Networks (CICSyN)

[5] Gomathi, E, Baskaran, K. "Recognition of Faces Using Improved Principal Component Analysis"; 2010 Second International Conference on Machine Learning and Computing (ICMLC)

[6] Haitao Zhao, Pong Chi Yuen" Incremental Linear Discriminant Analysis for Face Recognition", IEEE Transactions on Systems, Man, and Cybernetics, Part B: Cybernetics

[7] Tae-Kyun Kim; Kittler, J. "Locally linear discriminant analysis for multimodally distributed classes for face recognition with a single model image" IEEE Transactions on Pattern Analysis and Machine Intelligence, March 2005

[8] James, E.A.K., Annadurai, S. "Implementation of incremental linear discriminant analysis using singular value decomposition for face recognition". First International Conference on Advanced Computing, 2009. ICAC 2009

[9] Zhao Lihong, Wang Ye, Teng Hongfeng; "Face recognition based on independent component analysis", 2011 Chinese Control and Decision Conference (CCDC)

[10] Yunxia Li, Changyuan Fan; "Face Recognition by Non negative Independent Component Analysis" Fifth International Conference on Natural Computation, 2009. ICNC'09'.

[11] Yanchuan Huang, Mingchu Li, Chuang Lin and Linlin Tian. "GaborBased Kernel Independent Component Analysis on Intelligent Information Hiding and Multimedia Signal Processing (IIH-MSP).

[12] H.B.Kekre, Sudeep D. Thepade, Varun Lodha, Pooja Luthra, Ajoy Joseph, Chitrangada Nemani "Augmentation of Block Truncation Coding based Image Retrieval by using Even and Odd Images with Sundry Color Space" Int. Journal on Computer Science and Engg. Vol. 02, No. $08,2010,2535-2544$

[13] H.B.Kekre, Sudeep D. Thepade, Shrikant P. Sanas Improved CBIR using Multileveled Block Truncation Coding International Journal on Computer Science and Engineering Vol. 02, No. 08, 2010, 2535-2544

[14] H.B.Kekre, Sudeep D. Thepade, "Boosting Block Truncation Coding using Kekre"s LUV Color Space for Image Retrieval", WASET International Journal of Electrical, Computer and System Engineering (IJECSE), Volume 2, Number 3, pp. 172-180, Summer 2008.

[15] H.B.Kekre, Sudeep D. Thepade, "Image Retrieval using Augmented Block Truncation Coding Techniques", ACM International Conference on Advances in Computing, Communication and Control (ICAC32009), pp. 384-390, 23-24 Jan 2009, Fr. Conceicao Rodrigous College of Engg., Mumbai

[16] Developed by Dr. Libor Spacek. Available Online at: http://cswww.essex.ac.uk/mv/otherprojects.html

[17] Mark D. Fairchild, "Color Appearance Models", 2nd Edition, WileyIS\&T, Chichester, UK, 2005. ISBN 0-470-01216-1

[18] Rafael C. Gonzalez and Richard Eugene Woods "Digital Image Processing", 3rd edition, Prentice Hall, Upper Saddle River, NJ, 2008. ISBN 0-13-168728-X. pp. 407-413.S 
[19] Dr.H.B.Kekre, Sudeep D. Thepade and Shrikant P. Sanas, "Improved CBIR using Multileveled Block Truncation Coding", (IJCSE) International Journal on Computer Science and Engineering Vol. 02, No. 07, 2010, 2471-2476

[20] Dr. H.B.Kekre , Sudeep D. Thepade and Akshay Maloo, "Face Recognition using Texture Feartures Extracted from Walshlet Pyramid ”, Int. J. on Recent Trends in Engineering \& Technology, Vol. 05, No. 01, Mar 2011.

[21] International Journal on Biometrics and Bioinformatics, Volume 4 Issue 2 2010, http://www.slideshare.net/CSCJournals/international-journal-ofbiometrics-and-bioinformaticsijbb-volume-4-issue-2.

[22] Dr. H.B.Kekre, Sudeep D. Thepade, Varun Lodha, PoojaLuthra, Ajoy Joseph, Chitrangada Nemani,"Performance Comparison of Block Truncation Coding based Image Retrieval Techniques using Assorted Color Spaces", (IJCSIS) International Journal of Computer Science and Information Security, Vol. 8, No. 9,December 2010

\section{Authors PROFILE}

Dr. H. B. Kekre has received B.E. (Hons.) in Telecomm Engineering from Jabalpur University in 1958, M.Tech. (Industrial Electronics) from IIT Bombay in 1960, M.S.Engg. (Electrical Engg.) from University of Ottawa in 1965 and Ph.D. (System Identification) from IIT Bombay in $1970 \mathrm{He}$ has worked as Faculty of Electrical Engg. and then HOD Computer Science and Engg. at IIT Bombay. For 13 years he was working as a professor and head in the Department of Computer Engg. at Thadomal Shahani Engineering. College, Mumbai. Now he is Senior Professor at MPSTME, SVKM"s NMIMS University. He has guided $17 \mathrm{PhDs}$, more than 100 M.E./M.Tech and several B.E./B.tech projects. His areas of interest are Digital Signal processing, Image Processing and Computer Networking. He has more than 350 papers in National / International Conferences and Journals to his credit. $\mathrm{He}$ was Senior Member of IEEE. Presently He is Fellow of IETE and Life Member of ISTE Recently ten students working under his guidance have received best paper awards and two have been conferred Ph.D. degree of
SVKM'sNMIMS University. Currently 10 research scholars are pursuing Ph.D. program under his guidance.

Dr. Sudeep D. Thepade has Received B.E.(Computer) degree from North Maharashtra University with Distinction in 2003, M.E. in Computer Engineering from University of Mumbai in 2008 with Distinction, Ph.D. from SVKM"s NMIMS (Deemed to be University) in July 2011, Mumbai. He has more than 08 years of experience in teaching and industry. He was Lecturer in Dept. of Information Technology at Thadomal Shahani Engineering College, Bandra (W), Mumbai, for nearly 04 years. Currently working as Associate Professor in Computer Engineering at Mukesh Patel School of Technology Management and Engineering, SVKM"s NMIMS (Deemed to be University), Vile Parle (W), Mumbai, INDIA. He is member of International Advisory Committee for many International Conferences, acting as reviewer for many referred international journals/transactions including IEEE and IET. His areas of interest are Image Processing and Biometric Identification. He has guided five M.Tech. projects and several B.tech projects. He has more than 130 papers in National/International Conferences/Journals to his credit with a Best Paper Award at International Conference SSPCCIN-2008, Second Best Paper Award at ThinkQuest-2009, Second Best Research Project Award at Manshodhan 2010, Best Paper Award for paper published in June 2011 issue of International Journal IJCSIS (USA), Editor's Choice Awards for papers published in International Journal IJCA (USA) in 2010 and 2011.

Sanchit Khandelwal is currently pursuing B.tech. (CE) from MPSTME, NMIMS University, Mumbai. His areas of interest are Image Processing and Computer Networks and security. He has 1 paper in an international journal to his credit.

Karan Dhamejani is currently pursuing B.tech. (CE) from MPSTME, NMIMS University, Mumbai. His areas of interest are Image Processing, Computer Networks and UNIX programming. He has 2 papers in an international journal to his credit.

Adnan Azmi is currently pursuing B.tech. (CE) from MPSTME, NMIMS University, Mumbai. His areas of interest are Image Processing and Computer Networks. He has 1 paper in an international journal to his credit. 